\title{
THE MULTIDISCIPLINARY APPROACH TO MOOD DISORDERS
}

\author{
Francesca Falzon Aquilina ${ }^{1,2,3,4}$, Mark Agius ${ }^{5,6,7}$, Kriti Sharma ${ }^{1,2}$ \& Anton Grech ${ }^{4,8,9}$ \\ ${ }^{I}$ Foundation Year 2 Doctor, UK \\ ${ }^{2}$ Mater Dei Hospital, Msida, Malta \\ ${ }^{3}$ Mount Carmel Hospitals, Attard, Malta \\ ${ }^{4}$ Dar Kenn Ghal Sahhtek (Obesity \& Eating Disorders Services), Malta \\ ${ }^{5}$ Clare College Cambridge, The University of Cambridge, Cambridge, UK \\ ${ }^{6}$ South Essex Partnership University Foundation Trust, UK \\ ${ }^{7}$ Department of Psychiatry, University of Cambridge, Cambridge, UK \\ ${ }^{8}$ University of Malta, Malta \\ ${ }^{9}$ BCMHR-Cambridge University, Cambridge, UK
}

\begin{abstract}
SUMMARY
This article outlines the use of the multidisciplinary approach to mood disorders in the Short Stay Psychiatric Unit (SSPU) in Mater Dei Hospital, Msida, Malta.

An audit was carried out on all patients whose first admission to SSPU was in 2008 and who were diagnosed with having a mood disorder. The aim was to see whether the use of a multidisciplinary approach is in actual fact affecting patient outcome or not. The latter was measured by recording whether the patients needed any further admission and if this was the case, the length in days leading to their 2nd admission.
\end{abstract}

Key words: multidisciplinary approach - mood disorders - outcome - re-admission

Abbreviations: MDA - multidisciplinary approach, $\quad \mathrm{MCH}$ - Mount Carmel Hospital, MDH - Mater Dei Hospital, SSPU - Short Stay Psychiatric Unit

$* * * * *$

\section{INTRODUCTION}

The integrative approach to treatment of psychiatric patients incorporates a variety of therapeutic procedures that overlap and are not mutually exclusive.

Their objective is unique: to heal and to restore patients' proper functioning in their family, work and social environments alike. Treatment often requires integration of various therapeutic approaches (Ruzić 2010).

As a consequence, while community psychiatry developed in many countries in Europe from the 1960s, it developed in the form, particularly in the UK, of multidisciplinary teams, with members drawn from different specialities; doctors, nurses, psychologists, social workers, and Ocupational therapists. However, there was little done to prove that the use of a multidisciplinary approach involving several professions, provided any clinical advantage to the patients. Later, with the development of Early Intervention in psychosis teams, it was possible to test the hypothesis that an Assertive Early Intervention multidisciplinary team provided better outcomes than treatment as usual in a standard multidisciplinary community mental health team (Agius 2007, Craig 2004, Nordentoft 2006). However this begs the fundamental question as to whether the use of a multidisciplinary approach actually gives any specific advantage to the patient.

Given that now all patients who receive care in the community in the UK do so in community multi- disciplinary teams of one type or another, it is impossible to carry out a study in the UK to establish whether there is an advantage for patients if they are treated in a multidisciplinary team. However, in Malta community psychiatry has only recently been established. A new psychiatric ward was established in Mater Dei Hospital in 2008. When patients were discharged they either were followed up in outpatients or they were also offered upport from another professional. We elected to audit outcomes in the treatment of patients who were offered this support after having received a diagnosis in the ward of a chronic mood disorder. Such patients suffer from a relapsing remitting condition, so the outcome we elected to measure was the time which was taken before the patients relapsed.

The type of treatment considered in our audit was the following:

- Pharmacological therapy;

- Electroconvulsive therapy;

- Psychological therapy;

- Occupational therapy;

- Social worker involvement.

We decided that any patient who was offered three of these treatments, was deamed to have received a multidisciplinary approach, while patients who were assigned only one or two of these modalities of treatments, were deamed to have received 'treatment as usual' or, in fact, had not received a multidisciplinary approach. Since there 
was not a community mental health team, but patients were only offered three different modalities of treatment in the community, the effect of any enhanced outcome could only be attributed to the presence of different modalities of treatment simultaneously rather than the presence of a specific, trained team.

The aim was to discover whether a multidisciplinary approach was being used and whether this affected the clinical outcome of each patient.

\section{METHOD}

Data was collected from the official logbook in the SSPU at Mater Dei Hospital which includes the patient's demographic data, ICD-10 given to each patient and the number of admissions for each patient. This logbook dates back from the opening of the SSPU in the year 2008, when Mater dei hospital started offering service to the public, till present.

However, the group of patients utilized for this audit had to meet the following two criteria:

1) Diagnosed with chronic mood disorder;

2) 1st ever admission to an acute psychiatric facility was at SSPU in 2008.

An excel spreadsheet was set up which included patients' details, ICD-10s, gender and modality/ies of treatment used. The group of patients were diagnosed with having either bipolar or unipolar depression and these weren't further segregated. Also, for a patient to be considered as having been given multidisciplinary care he/she must have had at least three or more modalities of treatment enlisted above.

In this way, two groups of patients were created. Group 1 was treated by means of multidisciplinary care and Group 2 didn't.

The outcome was measured by recording:

- the number of patients from both groups, whose 1st re-admission was to SSPU or $\mathrm{MCH}$;

- the duration in days between discharge from SSPU and 1st re-admission to SSPU or MCH.

In this way we were able to compare whether the use of the multidisciplinary approach actually prevented further admission or extended the time till the 2nd admission.

One should note that the location for the 2nd admission considered could have either been at SSPU or at the only other acute mental health service in Malta ie: Mount Carmel Hospital, Malta.

\section{RESULTS}

According to the above criteria, 87 patients were selected. These were divided into Group 1: in which *MDA was used and Group 2: in which MDA was not used. When MDA was used $50 \%$ of patients in group 1 where re-admitted while when MDA was not used 58\% of patients in group 2 were re-admitted. This gives a difference of $8 \%$ in following re-admissions (Figure 1-3).

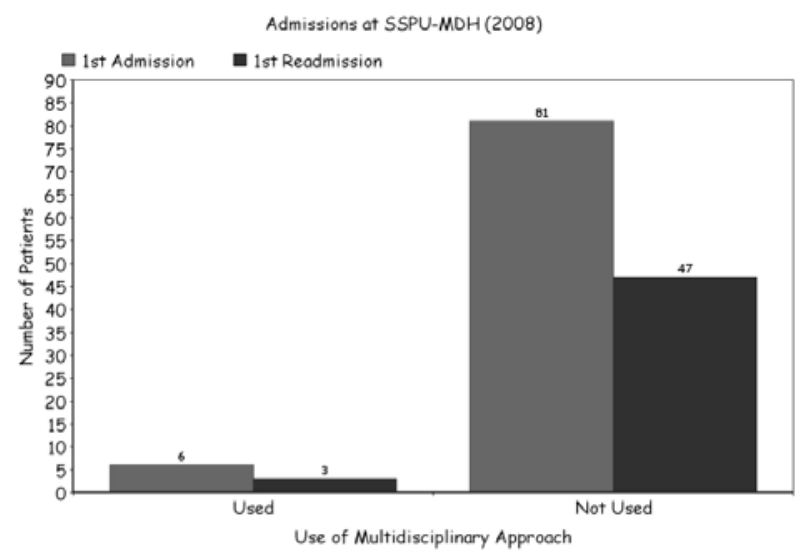

Figure 1. Above is a comparison of the number of readmissions occurring when MDA was used as opposed to when MDA was not used

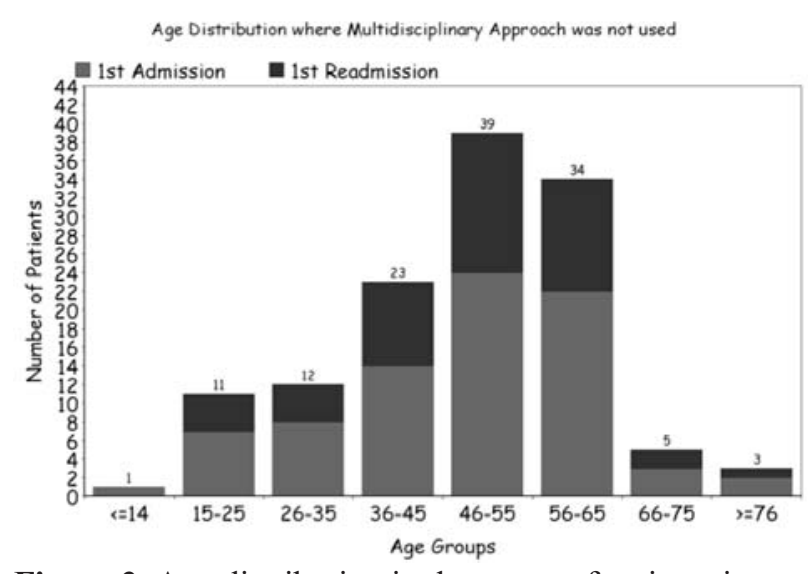

Figure 2. Age distribution in the group of patients in which MDA was used in both 1st admission and subsequent re-admission

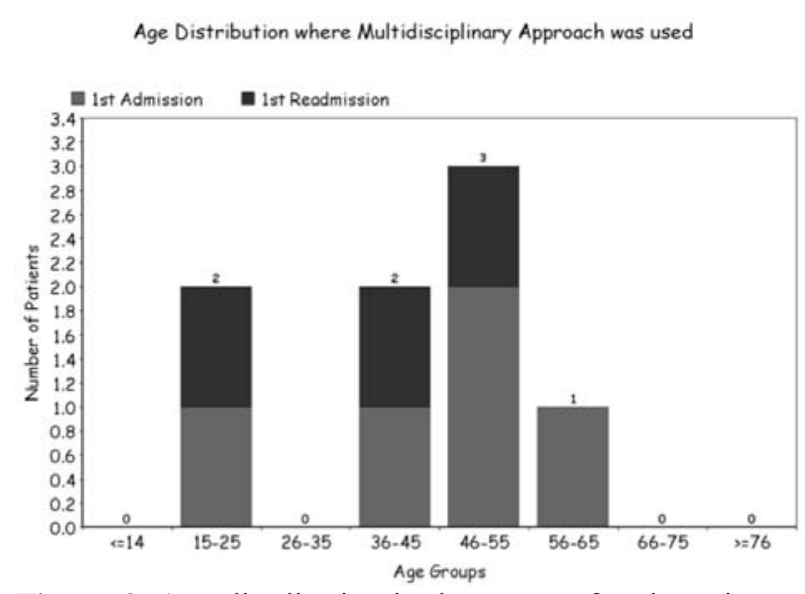

Figure 3. Age distribution in the group of patients in which MDA was not used in both 1st admission and subsequent re-admissions

Also, it was found that the average duration till next re-admission when MDA was used accounted to 561 days while when MDA was not used resulted in 480 days. Thus, this gives a difference of 81 days hospital stay. Each hospital bed at SSPU costs 300 euros daily, meaning that a total of 24,300 euros is saved when investing in multidisciplinary care for these patients. 


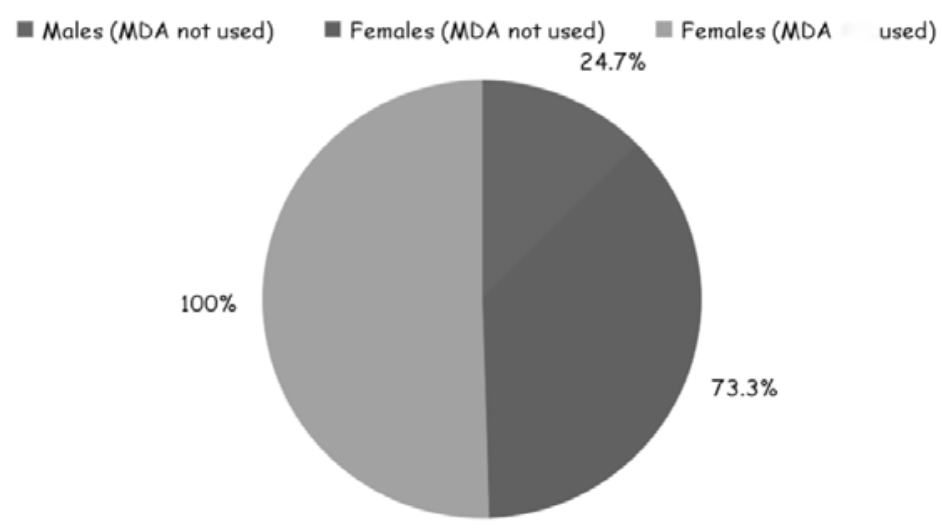

Figure 4. Gender distribution in the group where MDA was used (green) and MDA was not used (pink and purple according to gender)

It was also found that the vast majority of patients in both groups belong to the ages of 46-55 years.

In group $150 \%$ of patients belonged to that age group while in group 2 it was $48 \%$.

Moreover, as illustrated below the majority of patients belonged to the female gender, in both group 1 and group 2. In group one $100 \%$ of patients were females while in group two $73.3 \%$ belonged to this same gender (Figure 4).

\section{DISCUSSION}

This data demonstrated clearly that there was an improvement in the number of days between relapses of 81 days on average among the patients who received a multidisciplinary approach. This means that the hypothesis which we were testing has been proved. In our sample, patients who received a multidisciplinary approach remained well between relapses for a substantially longer period.

A number of studies have been carried out which have demonstrated that the use of a multidisciplinary team to follow up mood disorder patients, both in residential homes (Leontjevas 2013) and in community teams (Vieta 2005, Machado-Vieira 2004) have shown that relapses can be reduced, particularly if the intervention includes the identification of prodromal symptoms of relapse (Machado-Vieira 2004).

In our experience, the multidisciplinary approach to mood disorders is usually not used as first line management techniqe in most countries but our study clearly proved it significance. It plays a vital role in prolonging duration till first acute relapses especially in the age group 46-55.

This inreased incidence of mood disorders in the 46-55 age group correlates with other reports in the literature (Mojtabai 2004). Our data suggesting that most of the patients are female. This accords with the published literature. Though the American population showed a different trend compared to Europe but the most common gender remained female everywhere (NIMH 2012).
The NICE guidelines support the use of a multidisciplinary team in mood disorders since they recommend use of cognitive behaviour therapy or interpersonal therapy, which are as effective as antidepressant medications creating a clear foothold for a good multidisciplinary approach to be a part of guidelines in managing new and existing cases of mood disorders (Middleton 2005).

When assessing our patients, we found that the majority of patients being managed in a multidisciplinary environment had Psycotherapy, social worker, occupational therapy and pharmacotherapy as major modalities. There is evidence that each of the modalities we studied does mprove outcomes individually in the treatment of depression. ECT was occasionally noted as first line which according to nice guidelines is recommended as follows 'This should continue to be used, but its use should be restricted to achieving rapid and short term improvement in disabling symptoms in individuals with a severe depressive illness after other treatment options have proved ineffective or when the condition is potentially life threatening' (Middleton 2005). Hence the multidisciplinary modalities we used were also recommended to the NICE guidelines. No clear references exist about the use of social and occupational therapist involvement in NICE guidelines but a study published in pubmed states the significance of social worker involvement in women suffering from chronic depression and matrial discords (Corney 1984). Furthermore occupational therapy proved to be a modality used best whilst an inpatient with severe depression associated with suicidal tendencies (Custer 1991).

There are some limitations to our study. The group of patients who received multidiciplinary treatment was a much smaller group compared to those who did not. This is because few patients met our criteria in 2008 . Further research should examine a larger sample, and it is asumed that more patients received a multidisciplinary approach in later years. The study is a retrospective audit. It may be possible, in a country where multidisciplinary approach is still not usual, to carry out a prospective controlled study. 


\section{CONCLUSION}

It appears from our study that a multidisciplinary approach of itself increases the time between relapses in Mood Disorders.

\section{Acknowledgements: None.}

\section{Conflict of interest: None to declare.}

\section{References}

1. Agius M, Shah S, Ramkisson R, Murphy S, Zaman R: Three year outcomes of an Early Intervention For Psychosis Service as compared with treatment as usual for first psychotic episodes in a standard Community Mental Health Team-Final Results. Psychiatr Danub 2007; 19:130-138.

2. Corney RH: The effectiveness of attached social workers in the management of depressed female patients in general practice. Psychol Med Monogr Suppl 1984; 6:1-47.

3. Craig TK, Garety P, Power P, Rahaman N, Colbert $S$, Fornells-Ambrojo M, Dunn G: The Lambeth Early Onset (LEO) Team: randomised controlled trial of the effectiveness of specialised care for early psychosis. BMJ 2004; 329:1067.

4. Custer VL, Wassink KE: Occupational therapy interven tion for an adult with depression and suicidal tendencies. Am J Occup Ther 1991; 45:845-8.

5. Leontjevas $R$, Gerritsen $D L$, Smalbrugge M, Teerenstra $S$, Vernooij-Dassen M, Koopmans R: A structural multidisciplinary approach to depression management in nursing-home residents: a multicentre, stepped-wedge cluster-randomised tria. The Lancet 2013, 381:2255-2264.

6. Machado-Vieira $R$, Santin A, Soares JC: The multidisciplinary team approach to the treatment of bipolar disorder: an overview. Rev Bras Psiquiatr 2004; 26(Suppl 3):51-53.

7. Middleton H, Shaw I, Feder G: NICE guidelines for the management of depression. BMJ 2005; 300:267-268.

8. Mojtabai R, Olfson M: Major depression in communitydwelling middle-aged and older adults: prevalence and 2and 4-year follow-up symptoms. Psychol Med 2004; 34:623-34.

9. Nordentoft M1, Thorup A, Petersen L, Ohlenschlaeger J, Melau M, Christensen TØ, Krarup G, Jørgensen P, Jeppesen P: Transition rates from schizotypal disorder to psychotic disorder for first-contact patients included in the OPUStrial. A randomized clinical trial of integrated treatment and standard treatment. Schizophr Res 2006; 83:29-40.

10. Ruzić K, Knez R, Grahovac T, Dadić-Hero E, Graovac M: Integrative approach to treatment of a patient with psychotic depression. Psychiatr Danub 2010; 22:370-2.

11. Vieta E: The package of care for patients with bipolar depression. J Clin Psychiatry_2005; 66(Suppl 5):34-39.

12. http://www.nimh.nih.gov/statistics/1mdd_adult.shtml Major depression among adults.

Correspondence:

Dr. Francesca Falzon Aquilina M.D

Mater Dei Hospital

Msida, Malta

E-mail: francesca.falzon-aquilina@gov.mt 